\title{
EXISTENCE AND UNIQUENESS OF STANDARD BUBBLE CLUSTERS OF GIVEN VOLUMES IN $\mathbb{R}^{N *}$
}

\section{A. MONTESINOS AMILIBIA ${ }^{\dagger}$}

\begin{abstract}
We prove the existence of a unique standard $k$-bubble in $\mathbb{R}^{n}, k \leq n+1$, that encloses fixed volumes.

1. Introduction. A conjecture of much recent interest [SM, Prob. 2] says that a standard soap bubble cluster consisting of spherical surfaces meeting in threes at 120 degrees provides the least-perimeter way to enclose and separate $k$ regions of prescribed volume in $\mathbb{R}^{n}$ for $k \leq n+1$. Here we provide proofs of the existence and uniqueness of such a standard soap bubble.
\end{abstract}

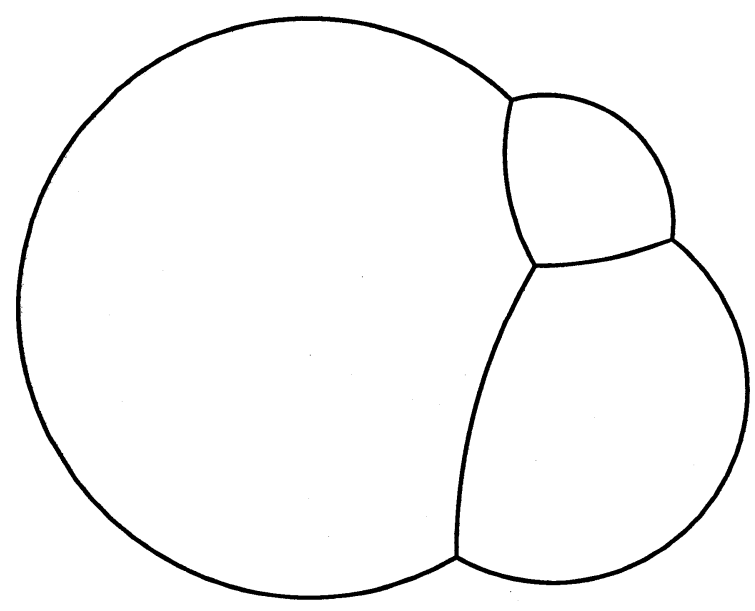

FIG. 1. Standard 3-bubble in $\mathbb{R}^{2}$

Progress on the conjecture. As for the conjecture itself, the case $k=1$ is the classical theorem that the round sphere provides the least-perimeter way to enclose given volumes in $\mathbb{R}^{n}$. The case $k=2$ is the Double Bubble Conjecture, proved in $\mathbb{R}^{2}$ in 1990 by a group of undergraduates [F2] and in $\mathbb{R}^{3}$ in 1995 for the case of equal volumes with the help of a computer by Hass, Hutchings, and Schlafly [HHS]. See [M1].

The case $k=3$, the Triple Bubble Conjecture, was proved for connected regions in $\mathbb{R}^{2}$ in 1992 by another group of undergraduates (including Hutchings) [C].

General results on the existence and regularity of minimizers are due to Almgren and Taylor ([A], [T], [AT]), Morgan [M2], and Hutchings [H, 2.6, 2.9, 5.1].

*Received June 19, 1999; accepted for publication August 31, 1999.

†Departamento de Geometría y Topología, Universidad de Valencia, Dr. Moliner 50, 46100 Burjasot (Valencia), Spain (montesin@uv.es). Work partially supported by the DGICYT (Spain) grant no. PB 91-0324. 
Earlier results on the existence and uniqueness of the standard bubble. Existence and uniqueness of the standard bubble has appeared for double bubbles in $\mathbb{R}^{2}$ and $\mathbb{R}^{3}$ [F2, Thm. 2.3], [F1, Thm. 3.2], and for the equal-area triple bubble in $\mathbb{R}^{2}$ [F1, Thm. 4.6]. Also Hutchings [H, p. 286], sketches a simple proof for double bubbles in $\mathbb{R}^{n}$ due to Morgan.

In $1995 \mathrm{~K}$. Brakke and also Hutchings proved the existence of standard $k$-bubbles in $\mathbb{R}^{n}(k \leq n+1)$. They used an idea of J. Sullivan's: obtaining a standard bubble as a stereographic projection of a regular simplex in the sphere.

This paper. Theorem 2.1 provides Brakke's beautiful proof of the existence of standard bubbles for given volumes. To prove uniqueness, it is more convenient to use radii than volumes as parameters. Proposition 3.2 proves existence (and uniqueness) for prescribed radii, using induction and an inversion which maps the spherical surfaces to hyperplanes, making it easy to insert an additional bubble at a vertex. Finally Theorem 4.2 deduces uniqueness for given volumes, using Lemma 4.1, which says that as one radius increases, the other volumes decrease.

Acknowledgments. I want to thank Frank Morgan for his helpful comments and for insisting that I write down these ideas. Also, to the referee that helped me a lot updating the status of the matter and suggested several improvements.

2. Existence of standard bubbles with prescribed volumes. For defining the standard bubble with more than one region we need to fix the angle at which two of its faces meet; thus, the dimension of these faces must be at least 1 and the bubble should live in $\mathbb{R}^{n}$ with $n \geq 2$. This will be assumed throughout the paper.

A standard $(n+1)$-bubble in $\mathbb{R}^{n}$ is a subset of $\mathbb{R}^{n}$ that is homeomorphic to the barycentric subdivision of an $n$-simplex; each of its faces (I mean always the $(n-1)$ dimensional ones) must be part of a sphere or a hyperplane and where two faces meet, they do at $120^{\circ}$ angles. The bubble divides $\mathbb{R}^{n}$ into $n+2$ regions. An inner interface separates two of the compact regions, and an outer face separates a compact region from the non compact one. We call center of the bubble the center of the barycentric subdivision, that is the point where all of the inner interfaces meet. A standard $k$ bubble in $\mathbb{R}^{n}, k \leq n$, is a subset homeomorphic to the result of removing an outer face from a $(k+1)$-bubble, and which satisfies the same conditions on faces and incidence angles as the $(n+1)$-bubble. We call outer or inner radii to the radii of the outer or inner faces.

Consider in $\mathbb{R}^{n+1}$ a regular $(n+1)$-simplex, $X$, and let $S$ be the sphere that passes through its vertices. From the center of $S$, project $X$ on $S$, and let $Y$ be that projection. By symmetry, where three of the projected $(n-1)$-dimensional faces meet, they must do at equal angles, that is at $120^{\circ}$ angles. Hence, the stereographic projection of $Y$ upon $\mathbb{R}^{n}$ gives a standard $(n+1)$-bubble. This is Sullivan's idea.

TheOREM 2.1. (Brakke[B]) Let $1 \leq k \leq n+1$ and let $V_{1}, \ldots, V_{k}>0$. Then there is a standard $k$-bubble in $\mathbb{R}^{n}$ that encloses and separates compact regions of volumes $V_{1}, \ldots, V_{k}$.

Proof. Look at a standard $k$-bubble in $\mathbb{R}^{n}$ as the stereographic projection from $S^{n}$ of the appropriate net dividing $S^{n}$ into $k+1$ regions, with the projection point in region $k+1$ that we denote $D$ (if $k \leq n$, intersect $D$ with the appropriate $S^{k-1}$ ). 
Note that $D$ is a simplex. Consider the map $f: D \rightarrow T$, where $T$ is the simplex in $\mathbb{R}^{k}$ that is the hyperplane $x_{1}+\cdots+x_{k}=1$ in the positive orthant, defined by

$$
f(P)=\frac{\left(\frac{1}{v_{1}}, \ldots, \frac{1}{v_{k}}\right)}{\frac{1}{v_{1}}+\cdots+\frac{1}{v_{k}}},
$$

where $v_{i}$ is the volume of region $i$ as projected from the point $P$. Then each $m$ dimensional face of simplex $D$ maps into a corresponding face of $T$, and by induction is a degree 1 map onto. $\square$

3. Existence of the standard bubble with prescribed outer radii. The standard 1-bubble in $\mathbb{R}^{n}$ is a sphere. The standard 2-bubble in $\mathbb{R}^{n}$ is built from two spheres that meet at $120^{\circ}$ angles and define the outer faces of the bubble, and a third sphere or hyperplane that meets them also at $120^{\circ}$ angles, as shown in Figure 2. The region enclosed by the outer faces is divided by the third hypersurface and in this manner we have the inner interface.

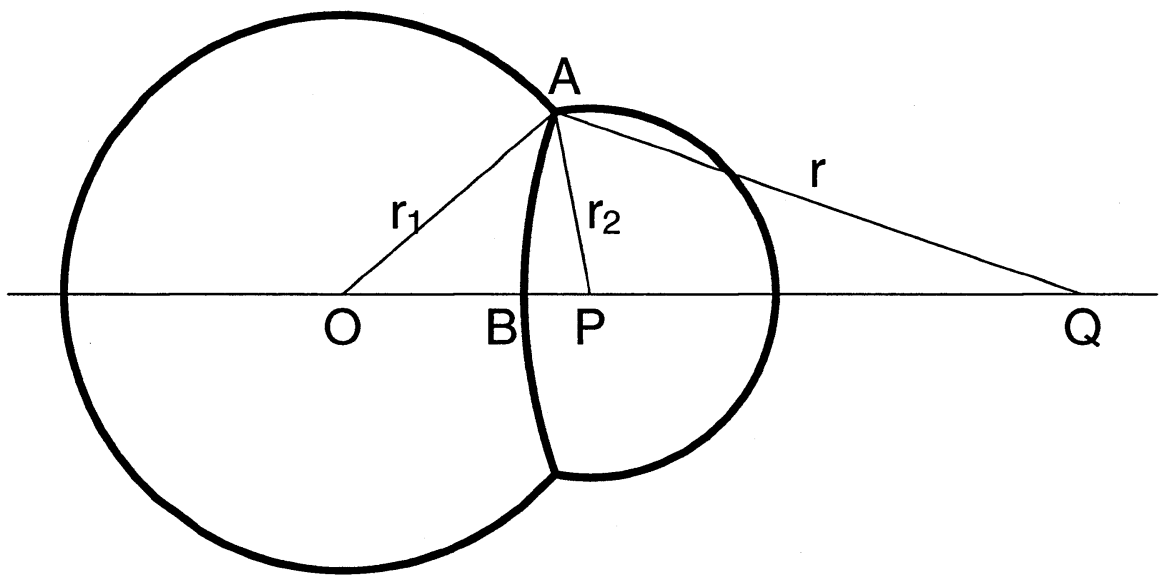

FIG. 2.

The following result will be useful.

Proposition 3.1. Let $\mathcal{O}, \mathcal{P}$ be two spheres in $\mathbb{R}^{n}$ with respective radii $r_{1}, r_{2}>0$. They meet at $120^{\circ}$ angles iff their centers are at a distance $\sqrt{r_{1}^{2}+r_{2}^{2}-r_{1} r_{2}}$. There is a unique standard 2-bubble in $\mathbb{R}^{n}$ whose outer faces have radii $r_{1}$ and $r_{2}$.

Proof. Figure 2 represents the intersection of the spheres with any 2-plane that passes through their centers. We see that the circles centered in $O$ and $P$ with respective radii $r_{1}$ and $r_{2}$ meet at $120^{\circ}$ angles iff the angle $\widehat{O A P}$ is $60^{\circ}$. That is iff $\cos (\widehat{O A P})=1 / 2$, that is iff $\overline{O P}^{2}=r_{1}^{2}+r_{2}^{2}-2 r_{1} r_{2} \cos (\widehat{O A P})=r_{1}^{2}+r_{2}^{2}-r_{1} r_{2}$, as claimed. Assume now that, for instance, $r_{1}>r_{2}$, and that both circles meet at $120^{\circ}$ angles. It is easy to see then that a circle that passes through $A$ and $A^{\prime}$ and therefore is centered (say, at $Q$ ) in the line $O P$ meets the other two circles at $120^{\circ}$ angles iff its radius is $r=r_{1} r_{2} /\left(r_{1}-r_{2}\right)$ and $P$ belongs to the segment $O Q$.

Proposition 3.2. Let $1 \leq k \leq n+1$ and $r_{1}, \ldots, r_{k}>0$. Then there is a unique (up to isometries of $\mathbb{R}^{n}$ ) standard $k$-bubble in $\mathbb{R}^{n}$ whose outer faces have radii $r_{1}, \ldots, r_{k}$. 
Proof. The uniqueness is obvious since, by 3.1, the distances among the centers of the outer faces are fixed so that these centers form a unique $(k-1)$-simplex, up to isometries; then, the positions of the centers of the inner interfaces, and their radii, are also fixed. However, for $k>2$ it is not obvious that we can always form a $(k-1)$-simplex with distances among vertices given by $\sqrt{r_{i}^{2}+r_{j}^{2}-r_{i} r_{j}}$.

First, assume that $2<k<n+1$, that $P$ is a $(k-1)$-plane in $\mathbb{R}^{n}$ and that $B_{k}$ is a standard $k$-bubble in $P$; the centers of the inner and outer faces of $B_{k}$ are at the appropriate distances according to their radii, so that we can define a standard $k$-bubble in $\mathbb{R}^{n}$ by 'blowing' $B_{k}$, that is the bubble built from the $(n-1)$-dimensional spheres with same centers and radii as the faces of $B_{k}$.

Now, we proceed by induction. Let $H$ be any hyperplane of $\mathbb{R}^{n}$, and $\tilde{B}_{n}$ a standard $n$-bubble in $H$ with outer radii $r_{1}, \ldots, r_{n}$. Let $B_{n}$ be the $n$-bubble in $\mathbb{R}^{n}$ obtained as before by blowing $\tilde{B}_{n}$. The intersection of all of the inner interfaces of $\tilde{B}_{n}$ is a point, so that the intersection of all of the inner interfaces of $B_{n}$ is an arc of circle (or line segment). Any of the ends, $A$ and $A^{\prime}$, of that arc or line segment belongs to all

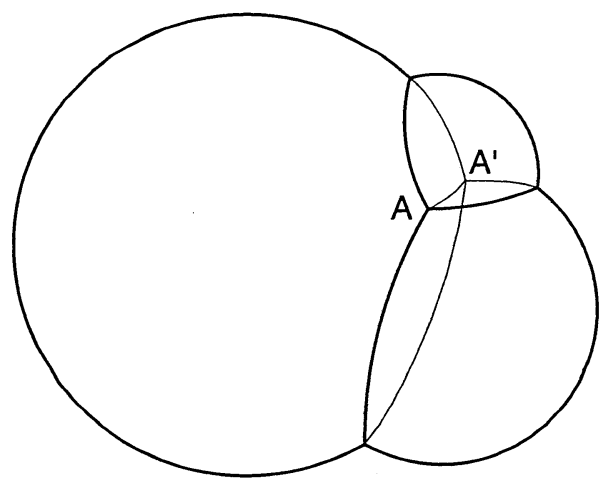

FIG. 3. Standard 3-bubble in $\mathbb{R}^{2}$ blown to $\mathbb{R}^{3}$

of the faces, inner and outer (see figure 3). Thus, a central inversion $\Phi$ with center $A$, for instance the one that leaves the other end, $A^{\prime}$, fixed, changes all faces of $B_{n}$ into parts of hyperplanes that meet at $A^{\prime}$ at equal angles. There are $n+1$ half-lines starting from $A^{\prime}$, lying as the ones connecting the center of a regular $n$-simplex with its vertices, such that each subset of $n-1$ of them determines the image of a face of $B_{n}$ under $\Phi$. Let us choose a point on each of these half-lines, all equidistant from $A^{\prime}$. With each of these points as center we consider the piece of sphere that passes through the other $n$ points and is contained inside the convex hull of the half-lines passing through those $n$ points (see figure 4); one of these convex hulls contains $A$ in its interior; let us call it $R_{A}$. Any pair of these spheres intersect at $120^{\circ}$ angles; also if $S$ is one of those spheres with center $c$ and $P$ is the image by $\Phi$ of a face of $B_{n}$ such that $c \notin P$, then $S$ and $P$ intersect also at $120^{\circ}$ angles. All this can easily be proved. All those spherical pieces enclose a region diffeomorphic to an $n$-simplex and we will assume that the choice of the centers of those spheres leaves $A$ outside that curved $n$-simplex. Its exterior is divided by the images of the faces of $B_{n}$ into another $n+1$ regions, and the image of these $n+2$ regions under $\Phi$ is thus a standard $(n+1)$-bubble. The image by $\Phi$ of the spherical piece that did lie in $R_{A}$ is the new outer face, and it is evident by continuity that we can give it any positive radius, for 


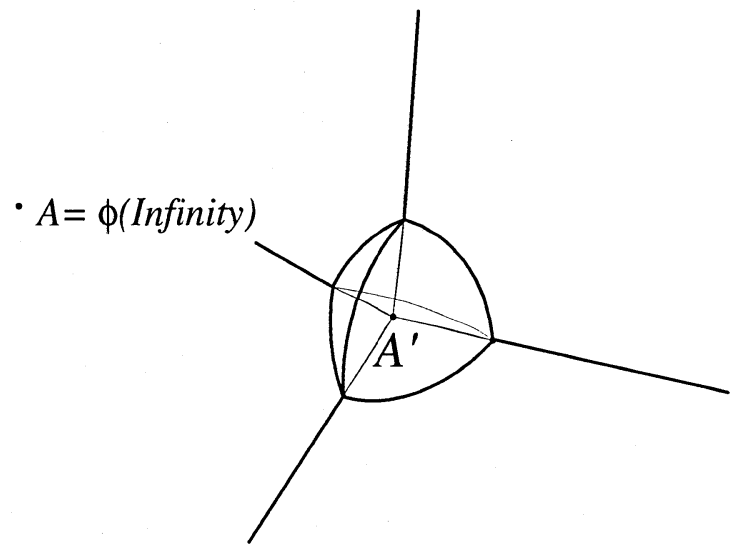

FIG. 4.

instance $r_{n+1} . \square$

4. Uniqueness of the standard bubble with prescribed volumes. Let $\mathbf{V}:\left(\mathbb{R}^{+}\right)^{k} \rightarrow\left(\mathbb{R}^{+}\right)^{k}$ be the function that to each set $r_{1}, \ldots, r_{k}$ of positive numbers assigns the volumes of the respective regions of the standard $k$-bubble in $\mathbb{R}^{n}, 1 \leq$ $k \leq n+1$, whose outer faces have radii $r_{1}, \ldots, r_{k}$. In order to prove the uniqueness of the standard $k$-bubble that encloses given volumes we need only to prove that $\mathbf{V}$ is injective. Let $\mathbf{V}_{1}, \ldots, \mathbf{V}_{k}$ be the components of $\mathbf{V}$.
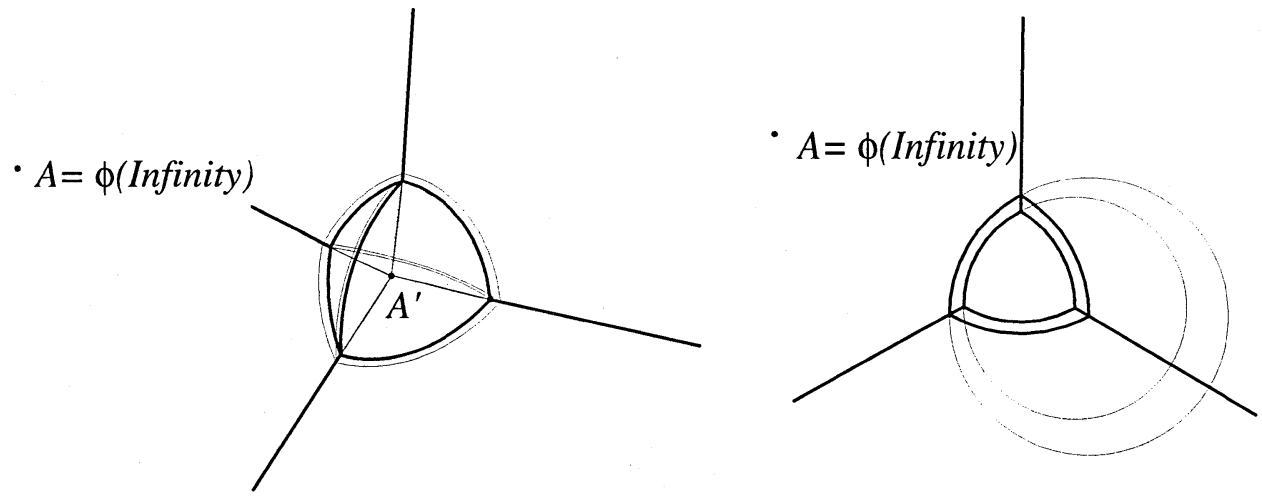

FIG. 5. Increasing the spherical caps in $\mathbb{R}^{3}$ and in $\mathbb{R}^{2}$

Lemma 4.1. Let $i \in\{1, \ldots, k\}$ and $r_{1}, \ldots, r_{k} \in \mathbb{R}^{+}$. Assume that $r_{i}^{\prime}>r_{i}$. Then

$$
\mathbf{V}_{j}\left(r_{1}, \ldots, r_{i}, \ldots, r_{k}\right)>\mathbf{V}_{j}\left(r_{1}, \ldots, r_{i}^{\prime}, \ldots, r_{k}\right)
$$

for $j \neq i$.

Proof. Let $B_{k}$ be a standard $k$-bubble in $\mathbb{R}^{k-1}$ with outer radii $r_{1}, \ldots, r_{k}$. According to the proof of 3.2 there is a point $A$ such that a central inversion $\Phi$ with center $A$ converts $B_{k}$ into a subdivision $C_{k}$ of $\mathbb{R}^{k-1}$ as described there (see figure 4). 
Since $\Phi^{2}=$ id and by $\Phi$ the point $A$ goes to infinity, the outer faces of $B_{k}$ are the images by $\Phi$ of the faces of $C_{k}$ that bound the region $R_{A}$ containing $A$. We can also assume that the $i^{\text {th }}$ outer face of $B_{k}$ is mapped into the spherical cap that forms part of the boundary of $R_{A}$. Let us increase the radius of all of these spherical pieces of $C_{k}$ (figure 5).

From the fact that the center of any of those spherical pieces is the opposite vertex we see that each of the complete spheres defining the new spherical caps of $C_{k}$ enclose completely the old corresponding complete spheres. The same must occur after mapping them by $\Phi$. Therefore, the new $i^{\text {th }}$ outer face of $B_{k}$ has greater radius that the former, and it is clear that this process can produce any previously specified radius $r_{i}^{\prime}$. Also, the remaining outer faces, being the images of the plane pieces bounding $R_{A}$, will have same radius as initially. The net result is, thus, a standard $k$-bubble with same outer radii except for the $i^{\text {th }}$ one, that has increased. But all of the regions of $C_{k}$, except the image of the $i^{\text {th }}$ region, have been reduced. The same will occur after inversion and this proves our claim, because obviously these inequalities are conserved after blowing $B_{k}$ to a standard $k$-bubble in $\mathbb{R}^{n}$.

THEOREM 4.2. The map $\mathbf{V}$ is injective. Thus, given $k$ positive volumes, there is (up to isometries of $\mathbb{R}^{n}$ ) a unique standard $k$-bubble in $\mathbb{R}^{n}$ that encloses those volumes.

Proof. It is clear that if $a \in \mathbb{R}^{+}$, then $\mathbf{V}\left(a r_{1}, \ldots, a r_{k}\right)=a^{n} \mathbf{V}\left(r_{1}, \ldots, r_{k}\right)$. Now, suppose that $\mathbf{V}\left(r_{1}, \ldots, r_{k}\right)=\mathbf{V}\left(r_{1}^{\prime}, \ldots, r_{k}^{\prime}\right)$. Then, let

$$
m=\max \left(\frac{r_{1}^{\prime}}{r_{1}}, \ldots, \frac{r_{k}^{\prime}}{r_{k}}, \frac{r_{1}}{r_{1}^{\prime}}, \ldots, \frac{r_{k}}{r_{k}^{\prime}}\right) .
$$

Clearly $m \geq 1$ and the equality sign implies $\left(r_{1}, \ldots, r_{k}\right)=\left(r_{1}^{\prime}, \ldots, r_{k}^{\prime}\right)$. Without loss of generality we can assume that

$$
m=\frac{r_{1}^{\prime}}{r_{1}}
$$

Then, we have

$$
\begin{gathered}
m^{n} \mathbf{V}_{1}\left(r_{1}^{\prime}, \ldots, r_{k}^{\prime}\right)=m^{n} \mathbf{V}_{1}\left(r_{1}, \ldots, r_{k}\right)=\mathbf{V}_{1}\left(m r_{1}, \ldots, m r_{k}\right) \\
=\mathbf{V}_{1}\left(r_{1}^{\prime}, m r_{2}, \ldots, m r_{k}\right) \leq \mathbf{V}_{1}\left(r_{1}^{\prime}, r_{2}^{\prime}, m r_{3}, \ldots, m r_{k}\right),
\end{gathered}
$$

by the Lemma, because

$$
m r_{2} \geq \frac{r_{2}^{\prime}}{r_{2}} r_{2}=r_{2}^{\prime}
$$

By iteration, we have

$$
m^{n} \mathbf{V}_{1}\left(r_{1}^{\prime}, \ldots, r_{k}^{\prime}\right) \leq \mathbf{V}_{1}\left(r_{1}^{\prime}, \ldots, r_{k}^{\prime}\right)
$$

Hence, $m \leq 1$ and $\mathbf{V}$ is injective, as claimed.

\section{REFERENCES}

[A] F. J. Almgren JR., Existence and Regularity Almost Everywhere of Solutions to Elliptic Variational Problems with Constraints, Memoirs AMS, vol. 4, no. 165, 1976. 
[AT] F. J. Almgren JR. AND J. E. TAYlor, Geometry of soap films, Sci. Amer., July, 1976, pp. 82-93.

[M1] Frank Morgan, Soap bubbles in $\mathbb{R}^{2}$ and in surfaces, Pac. J. Math, 165 (1994), pp. 347-361.

[C] Christopher Cox, Lisa Harrison, Michael Hutchings, Susan Kim, Janette Light, ANDrew MAuer, AND MEg Tilton, The shortest enclosure of three connected areas in $\mathbb{R}^{2}$, Real Anal. Exchange, 20 (1994/95), pp. 313-335.

[F1] Joel FoISY, Soap bubble clusters in $\mathbb{R}^{2}$ and $\mathbb{R}^{3}$, Williams College, undergraduate thesis, 1991, pp. 347-361.

[F2] Joel Foisy, Manuel Alfaro, Jeffrey Brock, Nickelous Hodges, and Jason Zimba, The standard double soap bubble in $\mathbb{R}^{2}$ uniquely minimizes perimeter, Pacific J. Math., 159 (1993), pp. 47-59.

[SM] John Sullivan ANd Frank Morgan (ed.), Open problems in soap bubble geometry, International J. of Math., 7 (1996), pp. 833-842.

[HHS] Joel Hass, Michael Hutchings, and Roger Schlafly, The double bubble conjecture, Elec. Res. Ann. Amer. Math. Soc., 1 (1995), pp. 98-102.

[H] Michael Hutchings, The structure of area-minimizing double bubbles, J. Geom. Anal., 7 (1997), pp. 285-304.

[M2] Frank Morgan, The Double Bubble Conjecture, FOCUS, Math. Assn. Amer., December, 1995.

[T] JEAN E. TAYLOR, The structure of singularities in soap-bubble-like and soap-film-like minimal surfaces, Ann. Math., 103 (1976), pp. 489-539.

[B] Ken Brakke, Personal communication, 1995. 